\title{
Caracterização de pessoas com diabetes em unidades de atenção primária e secundária em relação a fatores desencadeantes do pé diabético
}

Characteristics of people with diabetes regarding factors associated with the development of diabetic

foot ulcer

Caracterización de las personas con diabetes en unidades de atención primaria y secundaria en relación a factores desencadenantes del pié diabético

\section{Kattia Ochoa-Vigo', Maria Teresa da Costa Gonçalves Torquato ${ }^{2}$, Izilda Aparecida de Souza Silvério ${ }^{3}$, Flávia Alline de Queiroz $^{4}$, Mónica Cecilia De-La-Torre-Ugarte-Guanilo ${ }^{5}$, Ana Emilia Pace ${ }^{6}$}

\begin{abstract}
RESUMO
Objetivo: estudo descritivo que caracterizou pessoas com diabetes da rede básica de saúde em relação aos fatores desencadeantes do pé diabético, considerando variáveis socio-demográficas, história clínica e risco para complicações nos pés. Métodos: obtiveram-se dados mediante entrevista e avaliação dos pés. Na análise foi considerada avaliação dermatológica, estrutural, circulatória e neurológica e classificados conforme proposta de Sims e colaboradores. Resultados: a idade média foi 58,5 $\pm 8,5$ anos; tempo da doença 8,7 $\pm 6,7$ anos, com ensino fundamental incompleto. Nos antecedentes clínicos $73 \%$ referiram hipertensão arterial e 22\% dislipidemia. A glicemia média foi $159 \pm 79$ $\mathrm{mg} / \mathrm{dl}$ e $\mathrm{HbA}_{1 \mathrm{c}} 7,8 \pm 2 \%$. Na avaliação dos pés 73\% apresentaram higiene/corte de unhas impróprios, $63 \%$ pele ressecada/descamativa, 52\% unhas alteradas, $49 \%$ calos/rachaduras e $33 \%$ pé plano. Na classificação de risco identificou-se $7,9 \%$ com insensibilidade plantar e $6,9 \%$ com insensibilidade plantar e deformidade nos pés. Conclusões: estas alterações poderiam ser prevenidas com intervenções básicas e educação para cuidados com os pés.
\end{abstract}

Descritores: Diabetes mellitus; Pé diabético; Fatores de risco; Serviços básicos de saúde

\begin{abstract}
Objective: to describe factors associated with the development of diabetic foot ulcer among patients from primary health care settings regarding demographic variables, medical history, and risk for vascular complications. Methods: data were collected through interviews and feet examination, which consisted of dermatological, structural, circulatory, and neurological evaluation and classified according to Sims and colleagues. Results: the average age of the individuals with diabetes was $58.5 \pm 8.5$ years old, had diabetes for $8.7 \pm 6.7$ years, and had few years of elementary education. In terms of past medical history, $73 \%$ of the patients with diabetes also had high blood pressure and $22 \%$ had high cholesterol. They had an average blood glucose levels of $159 \pm 79 \mathrm{mg} / \mathrm{dl}$ and an average glycemic control $\left(\mathrm{HbA}_{1 \mathrm{c}}\right)$ of $7.8 \pm 2 \%$. Feet exam revealed that $73 \%$ of the patient had inadequate nail hygiene/trimming, $63 \%$ had dry/scaly skin, $52 \%$ had altered nail shape, $49 \%$ had corns/cracks, and 33\% had flat foot. Regarding vascular risk classification, $7.9 \%$ had plantar loss of sensitivity and $6.9 \%$ had both plantar insensitivity and feet deformity. Conclusion: the findings suggest that such alterations could be prevented through nursing interventions and feet care education.
\end{abstract}

Keywords: Diabetes mellitus; Diabetic foot ulcer; Risk factors; Primary care services

\section{RESUMEN}

Objetivo: estudio descriptivo que caracterizo a personas con diabetes de la red básica de salud en relación a los factores desencadenantes del pié diabético, considerando variables socio-demográficas, historia clínica y riesgo para complicaciones en los pies. Métodos: Se obtuvieron datos mediante entrevista y evaluación de los pies. En el análisis se consideró la evaluación dermatológica, estructural, circulatoria y neurológica y fueron clasificados de acuerdo a la propuesta de Sims y colaboradores. Resultados: la edad promedio fue de $58.5 \pm 8.5$ años; tiempo de la enfermedad $8.7 \pm 6.7$ años, con secundaria incompleta. En los antecedentes clínicos el $73 \%$ refirió hipertensión arterial y el 22\% dislipidimia. La glicemia promedio fue de $159 \pm 79 \mathrm{mg} / \mathrm{dl}$ y $\mathrm{HbA}_{1 \mathrm{c}} 7.8 \pm 2 \%$. En la evaluación de los pies el $73 \%$ presentó higiene/corte de uñas inapropiados, el 63\% piel reseca/descamada, el 52\% uñas alteradas, el 49\% callos/rajaduras y el 33\% pié plano. En la clasificación de riesgo se identifico el $7.9 \%$ con insensibilidad plantar y el $6.9 \%$ con insensibilidad plantar y deformidad en los pies. Conclusiones: estas alteraciones podrían ser prevenidas con intervenciones básicas y educación de cuidados con los pies.

Descriptores: Diabetes mellitus; Pié diabético; Factores de riesgo; Servicios básicos de salud

${ }^{1}$ Doutora da Posgraduação da Faculdade de Enfermagem da Universidad Peruana Cayetano Heredia - UPCH, Lima - Perú.

${ }^{2}$ Doutora em Clinica Médica, Assessora Técnica do Programa de Diabetes da Secretaria Municipal de Saúde de Ribeirão Preto (SP), Brasil.

${ }^{3}$ Docente da Faculdade SEMAR, Ribeirão Preto (SP), Brasil.

${ }^{4}$ Mestranda da Área de Enfermagem Fundamental da Escola de Enfermagem de Ribeirão Preto, Universidade de São Paulo - USP - Ribeirão Preto (SP), Brasil.

${ }^{5}$ Mestranda da Escola de Enfermagem, Universidade de São Paulo - USP - São Paulo (SP), Brasil.

${ }^{6}$ Doutora do Departamento de Enfermagem Geral e Especializada, Escola de Enfermagem de Ribeirão Preto da Universidade de São Paulo - USP - Ribeirão Preto (SP), Brasil. 


\section{INTRODUÇÃO}

No Brasil, estima-se que existem cinco milhões de habitantes com diabetes e metade deles desconhece o diagnóstico ${ }^{(1)}$. O estudo multicêntrico sobre prevalência do diabetes, realizado na faixa etária de 30 a 69 anos de idade, encontrou uma referência de $7,6 \%{ }^{(2)}$, e, na cidade de Ribeirão Preto - SP, foi de $12,1 \%{ }^{(3)}$; a doença atinge homens e mulheres da mesma forma, e é a quarta causa de morte. No Estado de São Paulo, a mortalidade por diabetes em maiores de 40 anos é superada apenas pelas doenças cardiovasculares ${ }^{(1)}$.

No geral, o diabetes é assintomático nos estágios iniciais, o que retarda seu diagnóstico durante anos ${ }^{(4)}$, aumentando o risco de complicações crônicas microvasculares, neuropáticas e macrovasculares, nos quais se destacam as doenças coronarianas, acidentes vasculares cerebrais e doenças vasculares periféricas, concomitante a maior probabilidade de desenvolver dislipidemia, hipertensão e obesidade ${ }^{(5)}$.

Adicionalmente, o diabetes mal controlado favorece o desenvolvimento de complicações altamente incapacitantes, principalmente, pé diabético, cegueira e insuficiência renal crônica, impedindo as pessoas de continuarem realizando suas atividades diárias e laborais, acarretando alta ocupação de leitos e absenteísmo ao trabalho, pelas internações prolongadas e recorrentes ${ }^{(6-7)}$.

O pé diabético representa um estado fisiopatológico multifacetado; caracterizado por úlceras que surgem nos pés da pessoa com diabetes e ocorrem como conseqüência de neuropatia em $90 \%$ dos $\operatorname{casos}^{(8)}$. As úlceras decorrem geralmente de traumas e se complicam com gangrena e infecção, ocasionados por falhas na cicatrização e podem resultar em amputação, quando não se institui tratamento precoce e adequado ${ }^{(8-10)}$.

Anualmente, de $2 \%$ a $3 \%$ das pessoas com diabetes podem desenvolver úlceras nos membros inferiores, e este percentual se eleva a $15 \%$ no transcurso de toda a sua vida. Pessoas com úlcera e/ou amputação prévia possuem importantes fatores de risco para recidivas ${ }^{(5)}$. As úlceras são responsáveis por grande percentual de morbimortalidade e hospitalização entre pessoas com diabetes $^{(11)}$, ocasionando um período de internação $59 \%$ mais prolongado que naquelas sem processos ulcerativos $^{(12)}$. Em geral, as hospitalizações por pé diabético são recorrentes e sua presença exige maior número de consultas ambulatórias e cuidados domiciliares $^{(13-14)}$.

Entre os casos graves hospitalizados, $85 \%$ são causados por úlceras superficiais, as quais apresentam comprometimento da sensibilidade protetora plantar, devido à neuropatia periférica, comumente associada a pequenos traumas, originados por objetos cortantes ao andar descalço(15), uso de calçados impróprios, dermatoses comuns, ou manipulações incorretas dos pés e unhas por pessoas não habilitadas ${ }^{(16)}$.

Considerando o prognóstico dos pacientes desenvolverem neuropatia periférica após longo período de sua instalação, em decorrência da demora no diagnóstico do diabetes. e reconhecendo sua contribuição relevante no processo de ulceração, este estudo objetivou caracterizar as pessoas com diabetes mellitus tipo 2, acompanhadas na rede de saúde municipal, em relação às variáveis socio-demográficas, história clínica e avaliação de risco para complicações nos pés, com a finalidade de identificar fatores desencadeantes para formação do pé diabético.

\section{MÉTODOS}

Estudo descritivo desenvolvido em duas Unidades Básicas Distritais de Saúde - UBDS - localizadas ao Norte e sul da cidade de Ribeirão Preto, no Estado de São Paulo, com prévia aprovação do Comitê de Ética e Pesquisa da Escola de Enfermagem de Ribeirão Preto - USP e anuência da Secretária Municipal de Saúde de Ribeirão Preto. A população foi constituída por pessoas com diabetes tipo 2, com idade acima de 40 anos, residentes nos respectivos bairros, identificadas nos dados informatizados da farmácia e no sistema HIGYA da Secretaria de Saúde em cada UBDS. Desta forma, 584 (213 no norte e 371 no sul) pessoas foram convocadas às respectivas unidades mediante carta, entre setembro e dezembro de 2003, em horários pré-estabelecidos.

No total compareceram 416 pessoas e, dessas, 35 não atenderam aos critérios de inclusão (diagnóstico de diabetes após 30 anos de idade, perfusão tissular preservada em membros inferiores, função renal preservada, raciocínio e juízo conservados) e 251 não mostraram interesse. Portanto, 101 pessoas foram entrevistadas e submetidas à avaliação de seus pés, em ambas as unidades.

O instrumento de coleta de dados foi semi-estruturado, elaborado a partir de estudo prévio ${ }^{(17)}$ e composto de duas partes. A primeira parte incluiu aspectos de identificação do participante, dados socio-demográficos, dados relacionados à doença e controle metabólico, antecedentes clínicos e de problemas nos pés. A segunda parte contemplou dados relacionados com avaliação dos membros inferiores, incluindo condição dermatológica, estrutural, circulatória e neuropática e características dos calçados por ocasião da entrevista. Esses dados permitiram identificar o grau de risco para lesão/ ulceração nos pés dos participantes, utilizando uma classificação de risco para pé diabético ${ }^{(18)}$, constituída por seis categorias, de zero a 5 , a qual inclui aspectos relevantes para intervenção precoce pela equipe de saúde primária.

A coleta de dados foi realizada por duas autoras do 
trabalho, em consultório de cada UBDS, após assinatura doTtermo de Consentimento Livre e Esclarecido conforme os principios éticos vigentes, procedendo-se, então, à entrevista e exame dos pés, com um tempo aproximado de 30-45 minutos, no qual também foram incluídas orientações sobre aspectos relevantes da doença e de seu controle, além de cuidados preventivos com os pés e calçados.

$\mathrm{Na}$ avaliação neurológica considerou-se a sensibilidade protetora plantar e a vibratória dorsal. $\mathrm{Na}$ sensibilidade protetora utilizou-se o monofilamento Semmes-Weinstein 5.07 (Sorri-Bauru, Brasil), o qual foi aplicado perpendicularmente em 10 pontos de cada pé (primeiro, terceiro e quinto dígitos plantar; primeira, terceira e quinta cabeça dos metatarsos plantar; lateral esquerda e direita do meio plantar; calcâneo e dorso entre primeiro e segundo dedos). Considerou-se sensibilidade comprometida, quando houve insensibilidade em quatro pontos, após três tentativas ${ }^{(19)}$. A sensibilidade vibratória foi testada utilizando diapasão de $128 \mathrm{~Hz}$ (Graham-Field Company, América do Norte) e aplicado, perpendicularmente, em quatro pontos de cada pé (maléolo interno e externo, dorsal média e distal falange do hálux). Considerou-se sensibilidade comprometida, quando houve insensibilidade em dois pontos, após três tentativas ${ }^{(20)}$.

$\mathrm{Na}$ avaliação dos calçados consideraram-se quatro características: estilo (modelo), largura, comprimento e material de fabricação. O estilo foi apropriado quando era fechado, preferencialmente, protegendo todo o pé; na largura e comprimento, considerou-se aquele de tamanho adequado, ou seja, nem grande, nem pequeno, com espaço de um centímetro a mais da anatomia do pé e, no material, este devia ser confeccionado em couro macio ou lona/algodão ${ }^{(21)}$. Portanto, o calçado foi apropriado quando as quatro características estiveram corretas.

Dados relacionados com controle metabólico foram solicitados aos participantes, quando estes não foram identificados nos seus respectivos prontuários, os quais foram realizados em Laboratórios de Referência conveniados para cada UBDS, conforme métodos disponibilizados neles. Desta forma, para a glicemia utilizou-se o método automatizado, para o colesterol total o método Enzimático Automatizado e Labetest, para HDL colesterol o método Precipitação Seletiva e Labetest, para as triglicérides o método Enzimático Calorimétrico e Automatizado e para creatinina, o método Jaffe Automatizado. Considerando existência de variabilidade de métodos entre laboratórios, os resultados obtidos foram equiparados para apresentação no estudo. A hemoglobina glicosilada $\left(\mathrm{HbA}_{1 \mathrm{c}}\right)$ foi realizada com o método de Cromatografia Líquida de Pressão Baixa de Troca Iônica (DiaSTAT ${ }^{\mathrm{TM}}$-Bio-Rad), com valor de referência de 4,3-6,4\%.

Para análise dos dados sangüíneos e da pressão arterial consideraram-se as metas propostas pela Sociedade Brasileira de Diabetes expressas no Consenso Brasileiro sobre Diabetes 2002 ${ }^{(22)}$. Quanto ao índice de masa corporal (IMC), utilizou-se a classificação apresentada no Manual de Hipertensão Arterial e Diabetes Mellitus ${ }^{(23)}$, em consonância com a Organização Mundial de Saúde de 1998.

Os dados foram digitados no Programa Excel e processados no EPIINFO utilizando testes da estatística descritiva.

\section{RESULTADOS}

Observa-se, na Tabela 1, uma população adulta, com idade média de 58 48,5 anos, com maior participação do sexo feminino $(71,3 \%)$, sendo que a grande maioria, no momento da entrevista, referiu conviver com parceiro(a) e que se encontrava em condição de aposentada/do lar.

Oitenta e sete por cento da população tinham ensino fundamental incompleto, sendo que 8,9\% eram iletradas, $10 \%$ apenas tinham cursado um ano de estudo e $53 \%$ tinham entre dois e quatro anos de estudo, condição que pode caracterizar a população de baixa escolaridade.

O tempo de doença foi em média de 8,9 $\pm 6,7$ anos, sendo que, aproximadamente, $19 \%$ vinham recebendo terapia farmacológica combinada e $28 \%$ insulina. Quanto às co-morbidades, identificou-se obesidade com IMC em média de $31,1 \pm 4,8 \mathrm{~kg} / \mathrm{m}^{2}$, hipertensão arterial $(73,3 \%)$, dislipidemia $(21,8 \%)$ e retinopatia diabética $(5,9 \%)$.

Entre os antecedentes de problemas nos pés, os participantes relataram, freqüentemente, rachaduras $(29,7 \%)$ e unha encravada $(19,8 \%)$, observando-se uma baixa proporção de lesões menores, tais como cortes, queimaduras e bolhas.

A Tabela 2 inclui dados sobre o controle metabólico, destacando sua importância na prevenção e identificação precoce de neuropatia e complicações microvasculares $^{(24)}$. Nesse sentido, observa-se uma glicemia elevada $(=159,7 \pm 79,1 \mathrm{mg} / \mathrm{dl})$, paralelo a uma $\mathrm{HbA}_{1 \mathrm{c}}$ também fora do recomendado $(=7,8 \pm 2 \%)$. Entretanto, outros valores sangüíneos encontram-se dentro dos limites esperados.

$\mathrm{Na}$ Figura 1 observa-se as características dos participantes em relação à avaliação dos pés, considerando alterações sugestivas de lesão/ulceração, nas quais se destacam a higiene e corte de unha impróprios (73,3\%), pele resecada/descamativa (53,4\%), unha espessada/farinácea/onicomicose (52,5\%), calos/ rachaduras $(49,5 \%)$, dentre as condições dermatológicas, e pé plano $(33,7 \%)$, dentre as estruturais. Outros dados 
compreendem referência de claudicação $(15,8 \%)$ e identificação de insensibilidade protetora plantar (14,9\%).

Tabela 1 - Características sócio-demográficas e clínicas dos participantes

\begin{tabular}{|c|c|c|c|}
\hline \multirow[b]{2}{*}{ Variáveis } & \multicolumn{3}{|c|}{$(\mathrm{n}=101)$} \\
\hline & $x$ & DP & $\mathrm{n} / \%$ \\
\hline Idade & 58 & $\pm 8,5$ & \\
\hline Mulheres & & & $72 / 71,3$ \\
\hline Estado Civil* & & & $74 / 73,3$ \\
\hline Ocupação** & & & $70 / 69,3$ \\
\hline Escolaridade*** & & & $88 / 87,1$ \\
\hline $\begin{array}{l}\text { Tabagismo - consumo mínimo } \\
3 \text { vezes/semana }\end{array}$ & & & $38 / 37,6$ \\
\hline Tempo de doença & 8,9 & $\pm 6,7$ & \\
\hline Tratamento com agente oral & & & $54 / 53,5$ \\
\hline $\begin{array}{l}\text { Tratamento com insulina }+ \\
\text { agente oral }\end{array}$ & & & $19 / 18,8$ \\
\hline Tratamento com insulina & & & $28 / 27,7$ \\
\hline $\mathrm{IMC} \mathrm{kg/ \textrm {m } ^ { 2 }}$ & 31,1 & $\pm 4,8$ & \\
\hline PAS $\mathrm{mmHg}$ & 138,2 & $\pm 21,2$ & \\
\hline PAD $\mathrm{mmHg}$ & 80,5 & $\pm 12,7$ & \\
\hline Hipertensão arterial & & & $74 / 73,3$ \\
\hline Dislipidemia & & & $22 / 21,8$ \\
\hline Doença isquêmica do coração & & & $2 / 2,0$ \\
\hline Apoplexia & & & $1 / 1,0$ \\
\hline Retinopatia diabética & & & $6 / 5,9$ \\
\hline Neuropatia diabética & & & $1 / 1,0$ \\
\hline Outras doenças & & & $29 / 28,7$ \\
\hline $\begin{array}{l}\text { Antecedentes de queimadura } \\
\text { nos pés }\end{array}$ & & & $3 / 3,0$ \\
\hline Antecedentes de bolha nos pés & & & $3 / 3,0$ \\
\hline Antecedentes de corte nos pés & & & $4 / 4,0$ \\
\hline $\begin{array}{l}\text { Antecedentes de rachadura nos } \\
\text { pés }\end{array}$ & & & $30 / 29,7$ \\
\hline $\begin{array}{l}\text { Antecedentes de unha } \\
\text { encravada }\end{array}$ & & & $20 / 19,8$ \\
\hline
\end{tabular}

Casado/amasiado*; aposentado/do lar**; entre 1-8 anos de escolaridade -Ensino Fundamental***

Tabela 2 - Características bioquímicas dos participantes

\begin{tabular}{lcc}
\hline Variáveis & $(\mathrm{n})$ & $\overline{(\boldsymbol{X} \pm \mathrm{DP})}$ \\
\hline Glicemia de jejum $(\mathrm{mg} / \mathrm{dl})$ & $(95)$ & $159,7 \pm 79,1$ \\
$\mathrm{Hb} \mathrm{A}_{1 \mathrm{c}}(\%)$ & $(85)$ & $7,8 \pm 2,0$ \\
Colesterol Total $(\mathrm{mg} / \mathrm{dl})$ & $(89)$ & $186,5 \pm 34,2$ \\
HDL Colesterol $(\mathrm{mg} / \mathrm{dl})$ & $(80)$ & $39,4 \pm 12,6^{*}$ \\
Triglicérides $(\mathrm{mg} / \mathrm{dl})$ & $(87)$ & $194,4 \pm 101,1$ \\
Creatinina $(\mathrm{mg} / \mathrm{dl})$ & $(68)$ & $1,0 \pm 0,9$ \\
\hline
\end{tabular}

Quanto aos calçados, 30,7\% foram considerados apropriados pelo fato de serem fechados, com ponta ampla/arredondada ou semi-abertos, protegendo grande parte da área dos pés, incluindo-se, nesta condição, os calçados esportivos.

Finalmente, apresenta-se na Tabela 3, a classificação de risco para pé diabético dos participantes, considerando comprometimento da sensibilidade protetora plantar, presença de deformidades estruturais nos pés, intensidade da pressão plantar, história de úlcera e fratura neuropática.
Tabela 3 - Classificação de risco para pé diabético dos participantes, conforme categorização proposta por Sims, Cavanagh e Ulbrecht

\begin{tabular}{|c|c|c|}
\hline \multirow[t]{2}{*}{ Descrição das categorias } &  & $(n=101)$ \\
\hline & & $(\%)$ \\
\hline Sensibilidade intacta/preservada & 0 & 85,1 \\
\hline Perda de a sensibilidade protetora plantar & 1 & 7,9 \\
\hline $\begin{array}{l}\text { Perda de a sensibilidade protetora plantar }+ \\
\text { deformidade ou aumento da pressão plantar }\end{array}$ & 2 & 6,9 \\
\hline $\begin{array}{l}\text { Perda de a sensibilidade protetora plantar }+ \\
\text { história de úlcera prévia }\end{array}$ & 3 & - \\
\hline $\begin{array}{l}\text { Perda de a sensibilidade protetora plantar }+ \\
\text { história de úlcera }+ \text { deformidade ou aumento } \\
\text { da pressão plantar }\end{array}$ & 4 & - \\
\hline Fratura neuropática & 5 & - \\
\hline
\end{tabular}

Ressalta-se que a intensidade da pressão plantar foi avaliada apenas pela observação da presença de calosidades, o que não pode ser levado em conta na distribuição desta classificação, entretanto, observou-se que aproximadamente $50 \%$ da população possuíam calosidades/rachaduras.

\section{DISCUSSÃO}

As características sócio-demográficas identificadas neste estudo diferem dos resultados obtidos em população assistida no nível terciário, com relação à idade, sexo, estado civil e escolaridade, no qual se observam médias e percentuais menores ${ }^{(17)}$. Entretanto, estudo realizado em Passos - Minas Gerais, entre pessoas com diabetes tipo 2, registradas em Programa de Assistência ao Diabético na rede básica, a proporção de participantes do sexo feminino foi semelhante, porém, $61,4 \%$ tinham ensino fundamental incompleto ${ }^{(25)}$.

A questão da escolaridade representa um fator comum entre pessoas com diabetes, tanto em estudos nacionais e internacionais, onde o maior percentual atingido possui baixo grau escolar ${ }^{(26)}$, constituindo-se em fator agravante para o desencadeamento de complicações crônicas, pela limitação do acesso às informações, devido ao possível comprometimento das habilidades de leitura, escrita e compreensão das atividades de educação para o autocuidado preventivo.

Outros fatores que podem interferir no autocuidado dessas pessoas constituem, por eles próprio, a idade avançada, o sexo e o estilo de vida, este último determinado pelas crenças e valores, além da condição econômica e de saúde no geral.

O hábito de fumar apresenta-se também diferente dos dados de prévios estudos realizados em amostras brasileiras ${ }^{(17,25-26)}$. Considerando que a doença 
aterosclerótica vascular periférica se manifesta precocemente entre pessoas com diabetes, e que o tabagismo constitui um fator agravante para a obliteração das artérias e arteríolas, é importante sensibilizar nesta população o abandono desta prática como medida de prevenção e estilo de vida saudável.

Em relação ao tempo da doença e tratamento, observa-se, que grande proporção dos participantes recebem insulina $(27,7 \%)$, seja isolada ou associada a agentes orais $(18,8 \%)$. Tratando-se de pessoas acompanhadas na rede primária e secundária, este resultado pode revelar o comprometimento progressivo do diabetes tipo 2. Estudo realizado em unidade terciária mostrou que, nesse nível, mais de $70 \%$ dos pacientes vinham recebendo insulina entre diabetes tipo 1 e 2 e $30 \%$ já apresentavam doenças microvasculares ${ }^{(17)}$.

Nos dados do controle metabólico identifica-se que a glicemia em jejum e $\mathrm{HbA}_{1 \mathrm{c}}$ encontram-se fora das metas consideradas apropriadas, situação que favorece a instalação e desenvolvimento das complicações crônicas e, conseqüentemente, do risco de neuropatia, um dos fatores preponderantes para o desencadeamento de lesão/ulceração nos pés. Existe evidência consistente de que qualquer melhora no controle da glicemia, mediante terapia intensiva, diminui o risco e progressão das complicações microvasculares e neuropáticas ${ }^{(5,24)}$.

Uma condição comum entre pessoas com diabetes constitui a prevalência de obesidade, sendo que esta característica representa, na maioria das vezes, um estado pré-mórbido. O estudo de prevalência do diabetes mellitus realizado em Ribeirão Preto em 1997, na população de 30-69 anos, identificou que sujeitos obesos apresentaram uma prevalência $2,5(22,6 \%)$ vezes maior de diabetes do que indivíduos não obesos ${ }^{(26)}$.

No presente estudo observa-se uma população predominantemente obesa, atingindo $57,4 \%$ dos participantes, sendo que $28,7 \%$ já se encontravam com sobrepeso. Esta condição é preocupante, devido ao aumento do risco de complicações cardiovasculares, o qual se torna ainda mais grave, quando associado ao diabetes, devendo-se tomar providências no cuidado desse grupo de pessoas.

Assim, cabe às entidades gerenciadoras de saúde buscar estratégias para ampliar o programa multiprofissional de prevenção da obesidade já existente na rede ${ }^{(2)}$ a fim de oferecer acesso a maior número de pessoas com diabetes, considerando as implicações e danos que a obesidade traz à saúde e à sociedade em geral.

Outras co-morbidades já instaladas nesta população constituem a hipertensão arterial e dislipidemia. Os valores médios da pressão arterial apresentam-se elevados, segundo a meta estabelecida para seu controle - menor de $120 / 80 \mathrm{~mm} . H g .{ }^{(22)}$. Existem evidências que um tratamento farmacológico agressivo da pressão arterial reduz casos de acidente vascular encefálico, mortes relacionadas com diabetes, doenças cardíacas, complicações microvasculares e perda visual entre pessoas com diabetes tipo $2^{(5)}$. Dessa forma, a terapêutica medicamentosa poderia ser revista pela equipe médica, a fim de diminuir os potenciais riscos presentes nesta população.

$\mathrm{Na}$ avaliação dos membros inferiores, os resultados mostram-se semelhantes aos apresentados em estudos prévios identificados entre pessoas assistidas no nível terciário ${ }^{(17,26)}$. As condições dermatológicas observadas constituem parâmetros importantes de avaliação utilizados pelo enfermeiro, no processo do cuidado, permitindolhe determinar as intervenções básicas e planejar as ações educativas, conforme necessidades dos pacientes identificadas nas consultas de enfermagem.

A instalação da neuropatia periférica no organismo compromete as fibras autonômicas, motoras e sensitivas. Deste modo, o dano às fibras autonômicas reduz ou suprime o suor nos pés, deixando-os secos e predispondoos a rachaduras e fissuras, além de produzir alterações arterio-venosas. Quanto às fibras motoras, estas contribuem com a atrofia e fraqueza dos pequenos músculos dorsais do pé, desencadeando desequilíbrio nos tendões flexores e extensores dos metatarsos, além de deformidades e alterações no modo de caminhar ${ }^{(8)}$.

Destaca-se que deformidades ósseas nas cabeças metatarsianas do antepé representam áreas de excessiva pressão na superfície plantar na fase da marcha. Em tal sentido, a região plantar é protegida por um coxim adiposo, o qual tem a função de dissipar as forças do peso corporal por todas as direções; deslocamentos ou atrofias nesses coxins, em conseqüência da neuropatia motora e autonômica, e da disfunção metabólica do diabetes, provocarão aumento da pressão nessa área. Se esta pressão for suficientemente elevada em uma região, poderá ocorrer qualquer perda da pele ou desenvolvimento de hiperqueratose/calosidades ${ }^{(27)}$ que, se associadas a perda da sensibilidade protetora plantar, poderá desencadear-se uma úlcera.

Deste modo, é necessário que as pessoas com diabetes conheçam este mecanismo, para assim tomarem ciência da importância de cuidar de seus pés, tanto com medidas de higiene, hidratação e proteção com calçados apropriados, quanto com a inspeção diária dos pés na procura de algum sinal de lesão, salientando-se a inspeção do interior dos calçados, antes de usá-los.

Se as condições dermatológicas dos pés estiverem comprometidas, qualquer prejuízo na pele ou nos pés pode progredir até instalar-se uma lesão grave, que pode atingir tecidos profundos, especialmente se associadas a deformidades estruturais e perda da sensibilidade a dor. Neste ponto, a propriedade dos calçados adquire 
relevância na função de proteger os pés de agentes lesivos externos. Entretanto, os próprios calçados constituemse, também, em agentes lesivos, quando extremamente estreitos, ou extremamente folgados.

Calçados apertados podem causar lesões no dorso dos dedos e nos lados do antepé. Dedos em garra ou martelo podem resultar em pressão sobre o dorso proximal interfalangiano dentro de calçados pouco profundos. Calçados apertados e de ponta fina aumentam a pressão nas laterais do antepé e podem produzir isquemia na pele, se este estresse for aplicado continuamente ${ }^{(18)}$. Por outro lado, calçados folgados podem criar áreas de atrito, que favoreceriam a formação de bolhas ou, inclusive, o ingresso de pequenos objetos no interior dos mesmos, na fase da marcha.

No estudo, grande parte da amostra usava, na entrevista, calçados abertos, de ponta fina ou sandálias de dedo. Estes dados demonstram que existe desconhecimento dessas pessoas sobre a importância dos calçados fechados na prevenção de lesões nos pés. Desta forma, é preciso salientar a necessidade da indicação de calçados fechados, tipo esportivos ou mocassim, evitando aqueles com costuras internas.

Apesar da população estudada ser classificada de baixo risco, conforme classificação utilizada ${ }^{(18)}$,reforçase a importância do monitoramento dessas pessoas em relação às complicações nos pés, principalmente ao considerar a baixa escolaridade, mau controle metabólico, alterações dermatológicas e características dos calçados.

A avaliação periódica estabelecida na unidade de saúde para o seguimento dessas pessoas em relação a essa classificação de risco, permitirá identificar, precocemente, alterações que podem conduzir ao desencadeamento de lesão/ulceração nos pés, face à multicausalidade dos fatores internos decorrentes da própria deterioração do diabetes e dos fatores externos presentes no meio ambiente. No estudo, 15\% da população possuíam insensibilidade plantar, e destes, $7 \%$ apresentavam deformidade estrutural nos pés, constituindo, portanto, pessoas em alto risco de desenvolver úlcera, devido à formação de pontos de alta pressão.

Um estudo realizado em uma clínica ambulatorial, utilizando a categorização de Coleman ${ }^{(28)}$, incluindo 55 pacientes sem registro no prontuário de complicação nos pés identificou que $20 \%$ tinham neuropatia, $8 \%$ neuropatia mais deformidade e $40 \%$ apenas deformidade. Entretanto, $32 \%$ apresentaram neuropatia, deformidade mais comprometimento vascular, determinado pelo índice tornozelo- braquial. Os autores referem que $60 \%$ dessa população encontrava-se em grave risco de desenvolver pé diabético, considerando que o conhecimento sobre as condutas de cuidados com os pés eram inadequados.

Outro estudo de tipo transversal, recentemente publicado $^{(29),}$ e que utilizou a classificação de Wagner, realizado em um hospital de nivel terciário em Camerum, incluindo 300 pacientes, identificou que 27,3\% apresentavam insensibilidade ao monofilamento e 17,3\% possuiam deformidade estrutural no pés. E, entre os pacientes que tiveram exame completo dos pés $(14 \%)$, $47 \%$ possuiam corte de unhas imprópio e $22 \%$ utilizavam calcados mal ajustados a seus pés, abertos e/ ou com fivelas.

Por outro lado, são confirmados uma vez mais, os fatores preditivos de úlcera nos pés em recente publicação do estudo realizado em Ambulatorio de um Centro Médico na América do Norte incluindo 1.285 pacientes com diabetes, destacando a $\mathrm{HbA}_{1 \mathrm{c}}$, pobre visão, ulcera e amputação prévias, insensibilidade ao monofilamento e onicomicose ${ }^{(30)}$.

Desta forma, o presente estudo reforça os achados da literatura em relação à necessidade de seguimento das pessoas com diabetes, principalmente nos setores da atenção primária e secundária de saúde, visando à prevenção e/ou identificação precoce das complicações nos pés, que constituem uma grave ameaça a vida e elevado custo sócio-econômico para a pessoa, família e sistema de saúde.

Enfatiza-se também que fatores externos identificados neste estudo poderiam ser resolvidos e monitorados com ações de baixa complexidade. O enfermeiro deveria assumir a responsabilidade de rastrear e monitorizar os fatores de risco sugestivos de pé diabético identificados na população estudada e, com o envolvimento de toda a equipe de saúde, reforçar as orientações de autocuidado e, principalmente, a manutenção de um bom controle metabólico ${ }^{(21)}$.

\section{CONCLUSÃO}

No presente estudo identificou-se fatores desencadenates de formação do pé diabético como higiene e corte de unhas impróprios $(73,3 \%)$, pele ressecada/descamativa $(63,4 \%)$, unhas espessas/aspecto farináceo/onicomicose $(52,5 \%)$, calos/rachaduras $(49,5 \%)$ e dermatite fúngica/micose interdigital $(20,8 \%)$.

Outros dados identificados incluem deformidade estrutural (hálux valgo em 12,9\%, dedos sobrepostos/ garras em 5,9\%), alteração circulatória (edema em 10,9\%, claudicação em 15,8\%) e comprometimento da sensibilidade plantar (sensibilidade protetora em 14,9\%).

O estudo também apontou para condições que podem agravar esses fatores como a obesidade, hipertensão arterial, dislipidemia e mau controle glicêmico. Foram observados, dessa maneira, fatores de risco modificáveis e controláveis envolvidos no 
desenvolvimento do pé diabético, o que reforça a importância da consolidação do programa de atenção à pessoa com diabetes e envolvimento da equipe na busca do controle metabólico e prevenção das complicações crônicas, com ênfase no cuidado de complicações nos pés.

\section{AGRADECIMENTO}

À Fundação de Amparo a Pesquisa do Estado de São Paulo - FAPESP, pelo apoio financeiro no desenvolvimento desta pesquisa. Ao Dr. Carlos Raia (in memorian) da Secretaria Municipal de Saúde de Ribeirão Preto/São Paulo, pela aprovação do projeto.

\section{REFERÊNCIAS}

1. Sociedade Brasileira de Diabetes. Consenso Brasileiro de Conceitos e Condutas para o Diabetes Mellitus: recomendações da Sociedade Brasileira de Diabetes para a prática clínica. São Paulo; 1997. 56 p.

2. Malerbi DA, Franco LJ. Multicenter study of the prevalence of diabetes mellitus and impaired glucose tolerance in the urban Brazilian population aged 30-69 yr. The Brazilian Cooperative Group on the Study of Diabetes Prevalence. Diabetes Care. 1992; 15(11):1509-16.

3. Torquato MTCG, Montenegro Júnior RM, Vianna LAL, Souza RAHG, Lanna CMM, Lucas JCB, et al. Prevalence of diabetes mellitus and impaired glucose tolerance in the urban population aged 30-69 years in Ribeirão Preto (São Paulo), Brazil. São Paulo Med J. 2003; 121(6): 224-30.

4. Harris MI, Klein R, Welborn TA, Knuiman MW. Onset of NIDDM occurs at least 4-7 yr before clinical diagnosis. Diabetes Care. 1992; 15(7):815-9.

5. American Diabetes Association. Clinical Practice Recommendations 2003. Diabetes Care. 2003; 26(Suppl 1):1-156.

6. Dagogo-Jack S. DCCT results and diabetes care in developing countries. Diabetes Care. 1995; 18(3):416-7.

7. Lessa I, Mendonça GAS, Teixeira MTB. Doenças crônicas não transmissíveis no Brasil: dos fatores de risco ao impacto social. Bol Oficina Sanit Panam. 1996; 120(5):389-413.

8. Pedrosa HC, Nery ES, Sena FV, Novaes C, Feldkircher TC, Dias $\mathrm{MSO}$, et al. O desafio do projeto salvando o pé diabético. Boletim Médico do Centro BD de Educação em Diabetes: Terapêutica em Diabetes. 1998; 4(19):1-10.

9. Bild DE, Selby JV, Sinnock P, Browner WS, Braveman P, Showstack JA. Lower-extremity amputation in people with diabetes. Epidemiology and prevention. Diabetes Care. 1989; 12(1):24-31.

10. Caputo GM, Cavanagh PR, Ulbrecht JS, Gibbons GW, Karchmer AW. Assessment and management of foot disease in patients with diabetes. N Engl J Med. 1994; 331(13):854-60.

11. Levin ME. Foot lesions in patients with diabetes mellitus. Endocrinol Metab Clin North Am. 1996; 25(2):447-62. Review.

12. Reiber GE. Epidemiologia das úlceras e amputações do Pé
Diabético. In: Bowker JO, Pfeifer MA. Levin e O’Neal o Pé Diabético. 6a ed. Rio de Janeiro: Di Livros; 2001. cap. 2. p. 1333.

13. Ramsey SD, Newton K, Blough D, McCulloch DK, Sandhu N, Reiber GE, Wagner EH. Incidence, outcomes, and cost of foot ulcers in patients with diabetes. Diabetes Care. 1999; 22(3):3827.

14. Harrington C, Zagari MJ, Corea J, Klitenic J. A cost analysis of diabetic lower-extremity ulcers. Diabetes Care. 2000; 23(9):1333-8.

15. Boulton AJ. The diabetic foot: from art to science. The $18^{\text {th }}$ Camillo Golgi lecture. Diabetologia. 2004; 47(8):1343-53. Epub 2004 Jul 28.

16. Gross JL. Detecção e tratamento das complicações crônicas do Diabete Melito: consenso brasileiro. Arq Bras Endocrinol Metab. 1999; 43(1):7-13.

17. Pace AE, Foss MC, Ochoa-Vigo K, Hayashida M. Fatores de risco para complicações em extremidades inferiores de pessoas com diabetes mellitus. Rev Bras Enfermagem. 2002; 55(5):51421.

18. Sims DS Jr, Cavanagh PR, Ulbrecht JS. Risk factors in the diabetic foot. Recognition and management. Phys Ther. 1988; 68(12):1887-902.

19. Armstrong DG. Loss of protective sensation: a practical evidence-based definition. J Foot Ankle Surg. 1999; 38(1):7980.

20. Grupo de Trabalho Internacional sobre Pé Diabético. Diretrizes práticas: abordagem e prevenção do pé diabético. Brasília (DF): Secretaria de Estado de Saúde do Distrito Federal; 2001.

21. Ochoa-Vigo K, Pace AE. Pé diabético: estrategias para prevenção. Acta Paul Enfermagem. 2005; 18(1):100-9.

22. Sociedade Brasileira de Diabetes. Consenso Brasileiro sobre Diabetes: Diagnóstico e Classificação do Diabetes Melito e Tratamento do Diabetes Melito tipo 2. Rio de Janeiro: Diagraphic; 2003.

23. Ministério da Saúde. Manual de hipertensão arterial e diabetes mellitus. Brasília: Secretaria de Políticas de Saúde, Ministério da Saúde; 2002.

24. Singh N, Armstrong DG, Lipsky BA. Preventing foot ulcers in patients with diabetes. JAMA. 2005; 293(2):217-28.

25. Rossi VEC, Pace AE. Perfil das pessoas com diabetes mellitus tipo 2 cadastradas no programa de assistência ao diabético de Passos - MG. Enfermagem Brasil. 2003; 2(2):104-9.

26. Muniz ECS, Rocha RM, Reis ML, Santos VLCG, Grossi SAA. Avaliação do risco de ulcerações nos membros inferiores em portadores de diabetes mellitus tipo 2. Rev Esc Enfermagem USP. 1999; 33(N Esp):180-90.

27. Sumpio BE. Foot ulcers. N Engl J Med. 2000; 343(11):787-93.

28. Meijer JW, Links TP, Smit AJ, Groothoff JW, Eisma WH. Evaluation of a screening and prevention programme for diabetic foot complications. Prosth Orthot Int. 2001; 25(2):132-8.

29. Ndip EA, Tchakonte B, Mbanya JC. A study of the prevalence and risk factors of foot problems in a population of diabetic patients in cameroon. Int J Lower Extrem Wounds. 2006; 5(2):83-8.

30. Boyko EJ, Ahroni JH, Cohen V, Nelson KM, Heagerty PJ. Prediction of diabetic foot ulcer occurrence using commonly available clinical information: the Seatle Diabetic Foot Study. Diabetes Care. 2006; 29(6):1202-7. 


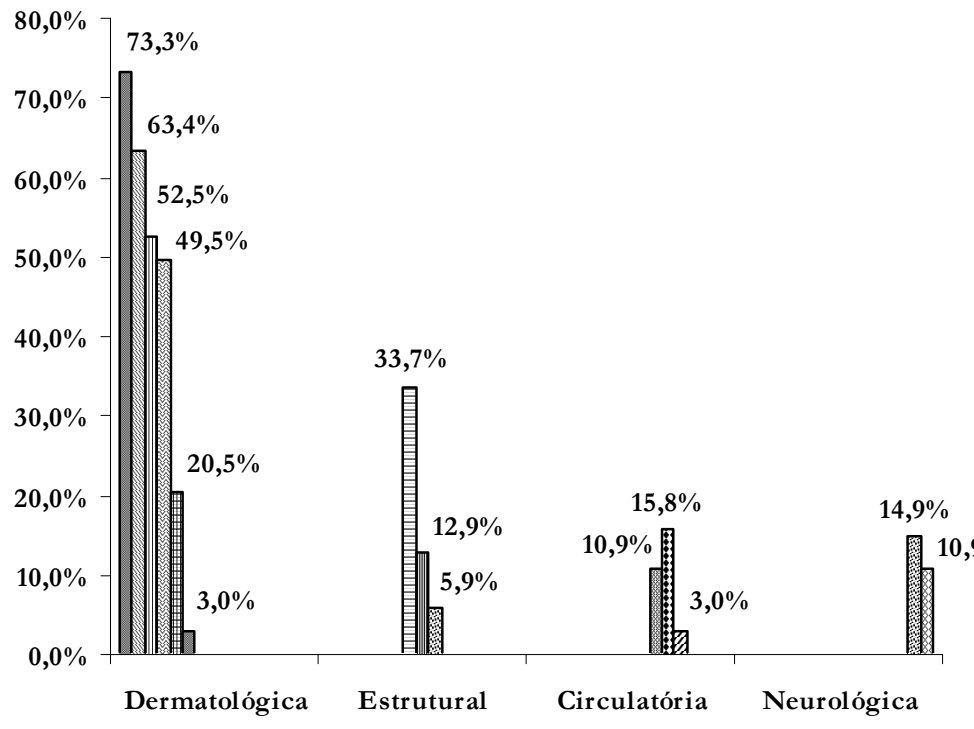

Figura 1 - Características dos pés identificadas ao exame nos participantes $\square$ Higiene dos pés e corte das unhas impróprios

৫ Pele ressecada/descamativa

四 Unha espessa/farinácea/onicomicose

圆 Calos/rachaduras

田 Dermatite fúngica/micose interdigital

口 Bolha/úlcera

目 Pé plano

四 Hálux valgo

图 Dedos sobrepostos

圆 Edema

由 Claudicação

๒ Pulso tibial posterior não palpável

国 Pulso pedioso não palpável

圈 Sensibilidade tátil pressórica plantar ausente

๑ Sensibilidade vibratória dorsal ausente 